\title{
Electron beam manipulation, injection and acceleration in plasma wakefield accelerators by optically generated plasma density spikes
} \author{
Jaroszynski $^{\mathrm{f}}$, G.G. Manahan ${ }^{\mathrm{f}}$, B. Hidding ${ }^{\mathrm{a}, \mathrm{f}}$ \\ ${ }^{a}$ Institute of Experimental Physics, University of Hamburg, 22761 Hamburg, Germany \\ ${ }^{b}$ Particle Beam Physics Laboratory, UCLA, Los Angeles, California 90095, USA \\ ${ }^{c}$ RadiaSoft LLC, Boulder, Colorado 80304, USA \\ ${ }^{d}$ RadiaBeam Technologies LLC, USA \\ ${ }^{e}$ Tech-X UK Ltd, Daresbury, Cheshire WA4 4FS, UK

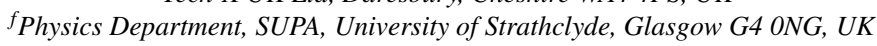

G. Wittiga , O. Karger ${ }^{\mathrm{a}}$, A. Knetsch ${ }^{\mathrm{a}}$, Y. Xi ${ }^{\mathrm{b}}$, A. Deng ${ }^{\mathrm{b}}$, J. B. Rosenzweig ${ }^{\mathrm{b}}$, D.L. Bruhwiler ${ }^{\mathrm{c}, \mathrm{d}}$, J. Smith ${ }^{\mathrm{e}}$, Z.-M. Sheng ${ }^{\mathrm{f}}$, D.A.

\begin{abstract}
We discuss considerations regarding a novel and robust scheme for optically triggered electron bunch generation in plasma wakefield accelerators [1]. In this technique, a transversely propagating focused laser pulse ignites a quasi-stationary plasma column before the arrival of the plasma wake. This localized plasma density enhancement or optical "plasma torch" distorts the blowout during the arrival of the electron drive bunch and modifies the electron trajectories, resulting in controlled injection. By changing the gas density, and the laser pulse parameters such as beam waist and intensity, and by moving the focal point of the laser pulse, the shape of the plasma torch, and therefore the generated trailing beam, can be tuned easily. The proposed method is much more flexible and faster in generating gas density transitions when compared to hydrodynamics-based methods, and it accommodates experimentalists needs as it is a purely optical process and straightforward to implement.
\end{abstract}

Keywords:

PACS: 52.40.Mj, 29.27.Ac, 52.50.Dg, 52.65.

\section{Introduction}

The dynamics of electron injection in plasma wakefield ac- ${ }^{28}$ celerators is a main focus of research in the plasma acceler- ${ }_{30}$ ator community, both experimentally and theoretically. The ${ }_{31}$ quality of the extracted witness bunches strongly depends on the process of trapping in the plasma wave. Several injection techniques have been proposed and have partially been demon- ${ }_{34}$ strated in LWFA as well as in PWFA such as [2, 3, 4, 5, 6, 7, 8, ${ }_{35}$ $9,10,11,12,13,14,15,16]$ as well as in the form of hybrid Trojan Horse-type methods [17, 18, 19, 20, 21, 22]. The "plasma torch" technique, as recently introduced in [1], is a flexible and simple technique for injection and trapping of electron bunches ${ }_{39}$ into the accelerating phase of plasma wakefield accelerators ${ }_{40}$ (predominantly, for PWFA, but potentially also for LWFA) and ${ }_{41}^{40}$ exploits a combination of optically generated density transitions as well as ionization injection and localized blowout am- ${ }_{43}$ plification effects.

Here, a focused laser pulse propagating perpendicularly (or at ${ }_{45}^{44}$ an arbitrary angle) to the driver beam axis ( $\mathrm{z}$ ) is used to ionize ${ }_{46}$ homogeneously distributed gas media in advance of the drive ${ }_{47}$ beam arrival, leading to a sharply spiked plasma density profile where the ionization threshold is exceeded by the electric ${ }_{49}$ field of the laser pulse. We refer to this region of optically excited, shapable plasma density volume as "plasma torch." This optical torch also has potential application in shaping of plasma cell boundaries and in the realization of ultrafast elec- tron bunch kickers [23]. The density elevation may be created on fs to many ps and up to ns time scale before the arrival of the electron-driven wakefield, using a modestly intense laser pulse, e.g. at the $I \sim 10^{15} \mathrm{~W} / \mathrm{cm}^{2}$ level in case of Ti:Sapphire laser pulses. The shape of the density profile is tunable by directly controlling parameters such as the energy and intensity profile of the laser pulse. Furthermore, this method does not require hydrodynamic expansion after optical excitation by a near-relativistic intense laser pulse, which is the prerequisite of the laser-driven (LWFA) scheme as discussed in Ref. [24, $5,25]$. In the plasma torch scheme, the plasma density shape does not rely on motion of ions and is a direct imprint of the applied laser profile and intensity, therefore very steep density transitions can be created on fs time scales. For example also between driver-witness electron bunch pairs even if they have few micrometer-scale distances, which may be useful to separate the drive beam from the witness. The plasma density spikes generated by the torch have ultrafast (fs-scale) rise times, and decay times of the order of the recombination timescale. The electron beam drives the plasma wave which is based on a low ionization threshold (LIT) gas species, while another (or more) gas component, which needs a higher ionization threshold (HIT), is left unaffected.

Both using a laser pulse at LIT or HIT ionization threshold intensities can be used to manipulate the beam-plasma interaction, including triggering injection of electrons, but the purely 
LIT-based plasma torch is limited to self-ionized PWFA cases 107 because in the preionized cases the LIT medium is preionized ${ }_{108}$ per definition and the plasma torch laser does not make any dif- ${ }_{109}$ ference at these intensities unless it additionally ionizes the HIT ${ }_{110}$ level.

\section{Trapping and acceleration of electron bunches for differ- ${ }^{11}$ ent plasma compositions and laser intensities}

Three possible scenarios of electron bunch trapping via the ${ }^{116}$ plasma torch scheme, supported by three dimensional particle- ${ }^{117}$ in-cell VSim/VORPAL[26] simulations, are considered. The ${ }^{118}$ plasma torch approach requires - as all PWFA schemes - an ${ }^{119}$ electron beam that can create a high-gradient plasma wake to ${ }^{120}$ trap electrons, while its electric fields must not ionize the HIT ${ }^{121}$ component. This is experimentally possible using a large range ${ }^{122}$ of electron beams, including those generated in LWFA stages. ${ }^{123}$ Here we use a FACET-class electron beam [27], having the fol-124 lowing parameters: charge $Q=3 \mathrm{nC}$, energy $E=23 \mathrm{GeV},{ }^{125}$ energy spread $\Delta E / E=2 \%$, bunch length $\sigma_{\mathrm{z}}=27 \mu \mathrm{m}$, trans- ${ }^{-126}$ verse size $\sigma_{\mathrm{r}}=8.5 \mu \mathrm{m}$, and normalized emittance $\epsilon_{\mathrm{n}}=2.25^{127}$ mm-mrad. A mixture of hydrogen and helium is implemented ${ }^{128}$ as the plasma source, where hydrogen with its low ionization ${ }^{129}$ energy is the LIT component and helium is the HIT compo-130 nent. Using the formula for the tunneling ADK rates [28], $\mathrm{a}^{131}$ peak field in the range of $\sim 90 \mathrm{GV} / \mathrm{m}$ is required to quickly ion-132 ize helium, which is hardly achievable even by a FACET-class ${ }^{133}$ electron beam. Therefore He will generally stay in the neutral ${ }^{134}$ state as long as the plasma torch laser does not ionize it pur-135 posefully

The use of a hydrogen/helium gas mixture allows for three qual-137 itatively different possible main scenarios: (i) initially hydro-138 gen and helium are in the neutral state, and the electron beam ${ }^{139}$ driver ionizes hydrogen on axis, while the plasma torch laser ${ }^{140}$ pulse does pre-ionize hydrogen locally in front of the drive ${ }^{141}$ beam. Helium is left in neutral state throughout the process; ${ }^{142}$ (ii) same as case (i), i.e. no preionization of hydrogen but ${ }^{143}$ self-ionization by the drive bunch, but the torch laser ionizes ${ }^{144}$ both hydrogen and helium locally; and (iii) hydrogen is com-145 pletely preionized, for example by an on-axis laser pulse (fo-146 cused by a lens, an axicon or advanced diffractive optics), and ${ }^{147}$ the torch laser ionizes additional helium locally in the pathway ${ }^{148}$ of the drive beam. It shall be noted that by using diffractive ${ }^{149}$ optics, it may be possible to adjust the on-axis intensity pro-150 file of the preionization laser pulse such that an intensity spike ${ }^{151}$ is generated, which then acts to a similar effect as an indepen-152 dently tunable plasma torch laser, albeit without its flexibility. ${ }^{153}$ In all selected simulations, a laser pulse propagates perpendic-154 ular to the electron beam and generates the plasma torch ap-155 proximately 1 ps before the electron-beam driven plasma wave ${ }^{156}$ arrives - this is to save computational costs by keeping the sim-157 ulation window sufficiently small. In reality, it does not mat-158 ter much if the plasma torch laser pulse arrives few hundred ${ }^{159}$ femtoseconds or many picoseconds before the electron beam ${ }^{160}$ driven plasma wave, as long as neither recombination effects ${ }^{161}$ nor ion and hydrodynamic motion sets in. This is advantageous ${ }^{162}$ because it means that the requirements put on the synchronization between electron beam driver and plasma torch laser pulse can be easily met. In the considered cases, the plasma torch laser pulse is based on a Ti:sapphire laser system, with central wavelength of $\lambda=800 \mathrm{~nm}$ and a pulse duration (FWHM) of $\tau=64$ fs. The delay between the torch laser and the electron driver was carefully chosen in the simulation such that the optical plasma torch is allowed to build up before the arrival of the electron beam, while at the same time the simulation box window length is minimized.

All given densities are free electron densities (when ionized), and atomic densities (when in neutral state), because only single ionization occurs both are equal. The actual parameters for the three cases are chosen based on analytical calculations of ionization levels and yields. All simulations of electron trapping triggered by optical plasma torches are compared with simulations without a plasma torch to confirm that the trapping is solely due to the plasma torch density perturbation. The combinations of laser waist $w_{0}$ and dimensionless amplitude $a_{0}$ have been chosen in all scenarios such that the torch width is equal or greater than the plasma wavelength $\lambda_{\mathrm{p}}=2 \pi c \sqrt{\epsilon_{0} m_{e} / n_{e} e^{2}}$ within the plasma torch, in order to allow for the plasma wave to interact at increased density at least over one $\lambda_{p}\left(m_{e}\right.$ being the electron mass, $c$ speed of light in vacuum, $e$ electron charge, $n_{e}$ the electron density, and $\epsilon_{0}$ the vacuum permittivity). On the other hand, a compact torch allows for a rapid density transition. It is known that the downramp length should be shorter than the plasma skin depth $k_{\mathrm{p}}^{-1}=c / \omega_{\mathrm{p}}$ [29] for electron bunch injection in PWFA. This criterion is fulfilled in each case.

Figure 1 shows the injection process of the first scenario, where neutral hydrogen is used and the torch laser is only ionizing hydrogen locally in the drive beam's path. In this case, the drive beam has to self-ionize hydrogen outside of the plasma torch region in order to generate a plasma, which is only possible near the center of the bunch, where the peak fields are high enough to exceed the hydrogen ionization threshold. Consequently, further ahead in the drive bunch there is no plasma, because the electric fields are much lower due to the smaller density, and outside the plasma torch region, the front part of the drive beam is simply unused.

The effective ionization front determines the beginning of the plasma wave, which is shifted rapidly to the front of the drive beam when entering the preionized region produced by the torch laser. Additionally, during the passage of the torch, the wakefield is significantly amplified, as now more drive beam current is contributing to the excitation. When the plasma torch volume is left and the drive beam exits the locally preionized hydrogen plasma region, the blowout shifts back again, since hydrogen once more needs to be self ionized by the drive beam. This snapping back of the plasma wave results in trapping of electrons very effectively. It is remarkable to note that neither the hydrogen gas density nor the plasma wavelength are changed during this process, which is a fundamental difference to gas density downramp injection.

After $z \approx 5 \mathrm{~mm}$ of propagation $(\approx 4.6 \mathrm{~mm}$ behind the torch), the generated witness bunch with energies exceeding $100 \mathrm{MeV}$ 


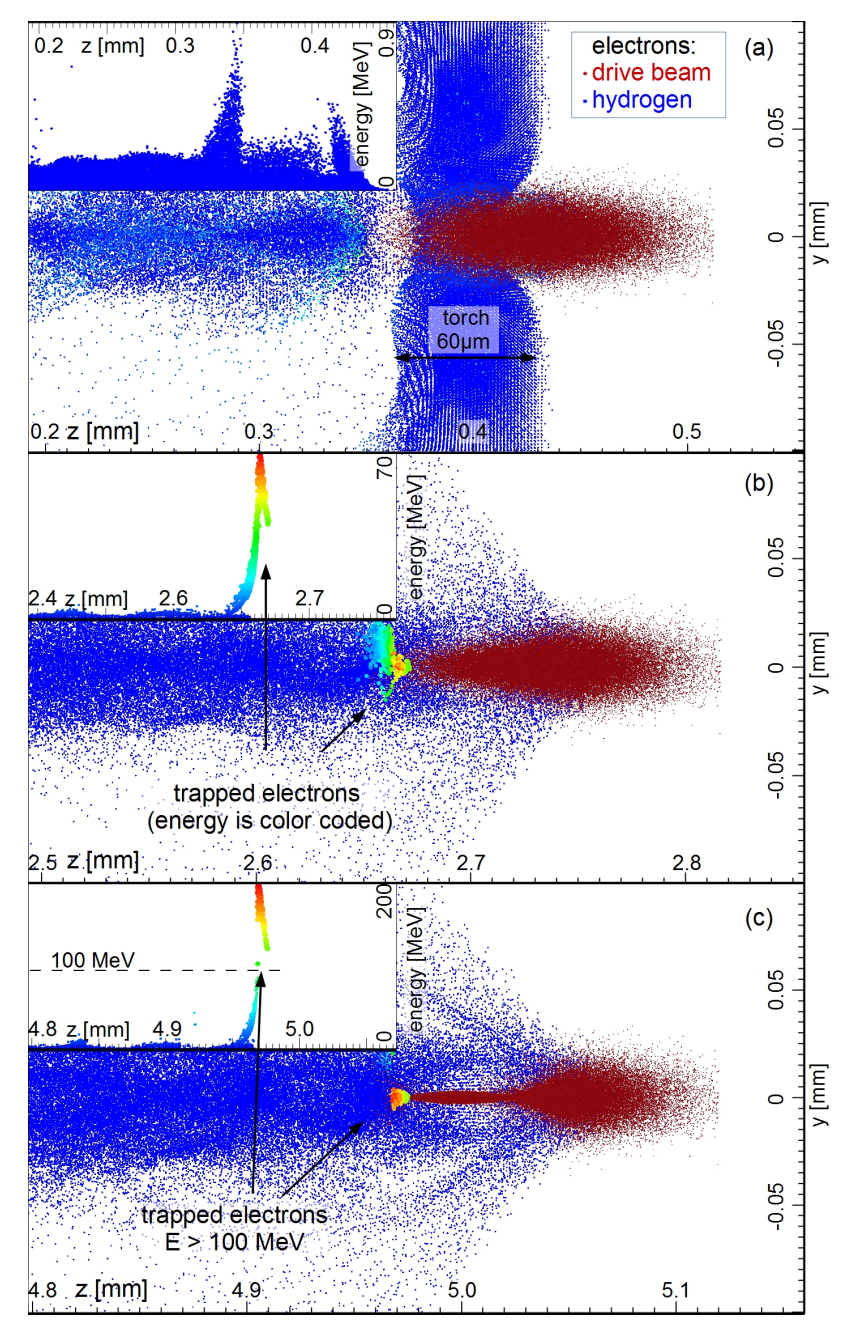

Figure 1: Optical plasma torch injection, illustrated by a central slice of a full $3 \mathrm{~d}$ simulation for scenario (i), using a hydrogen density of $n_{\mathrm{H}}=4 \times 10^{17} \mathrm{~cm}^{-3}$ ( $y, z$ are the torch laser, and drive beam propagation axes). The plasma torch is generated in the path of the drive beam, and crossed in (a), leading to blowout amplification and injection. Applying a laser pulse with $w_{0}=35 \mu \mathrm{m}$, and $a_{0}=$ 0.015 a torch of approximately $60 \mu \mathrm{m}$ width is created. The injected hydrogen electrons (color coded spheres) are shown in $(\mathrm{b}) \approx 2.2 \mathrm{~mm}$ after the torch, and in (c) after $\mathrm{z} \approx 4.6 \mathrm{~mm}$ of acceleration, where maximum energies of $\mathrm{E} \approx 194$ $\mathrm{MeV}$ are reached.

has normalized emittance of $\epsilon_{\mathrm{n}}=5.3 \mathrm{~mm} \mathrm{mrad}, Q=36 \mathrm{pC}, 179$ mean energy of $E=153 \mathrm{MeV}$, energy spread $\sigma_{E} / E=13.2 \%$; 180 and a peak current of $I_{\text {peak }}=2.5 \mathrm{kA}$. Also evident in Figure 1

(c) is the lensing effect of the plasma on the drive bunch which supports the wake's acceleration field strength.

In Figure 2 the second scenario is illustrated, similar to Fig-182 ure 1 . Here, the torch laser intensity is increased to $a_{0}=0.03^{183}$ in order to allow the local laser ionization of both helium and ${ }^{184}$ hydrogen. Obtained witness bunch parameters at $\mathrm{z} \approx 5 \mathrm{~mm}$, 185 counting electrons with energies exceeding $60 \mathrm{MeV}$ are: nor-186 malized emittance $\epsilon_{\mathrm{n}}=1.0 \mathrm{~mm}$ mrad; charge $Q=274 \mathrm{pC} ; 187$ mean energy $E=118 \mathrm{MeV}$; energy spread $\sigma_{E} / E=12.0 \%$; and ${ }^{188}$ a peak current at $I_{\text {peak }}=11.1 \mathrm{kA}$. Electrons of both elements ${ }^{189}$ are trapped and form the witness bunch. The total amount ${ }^{190}$ of trapped charge can be independently tuned via the helium ${ }^{191}$ gas density (up to beam loading levels) in contrast to scenario ${ }^{192}$ (i). In fact, here the accelerating filed is lowered, compared to case (i), due to the much higher trapped charge and consequently the energy gain is decreased. In Figure 3 the last sce-

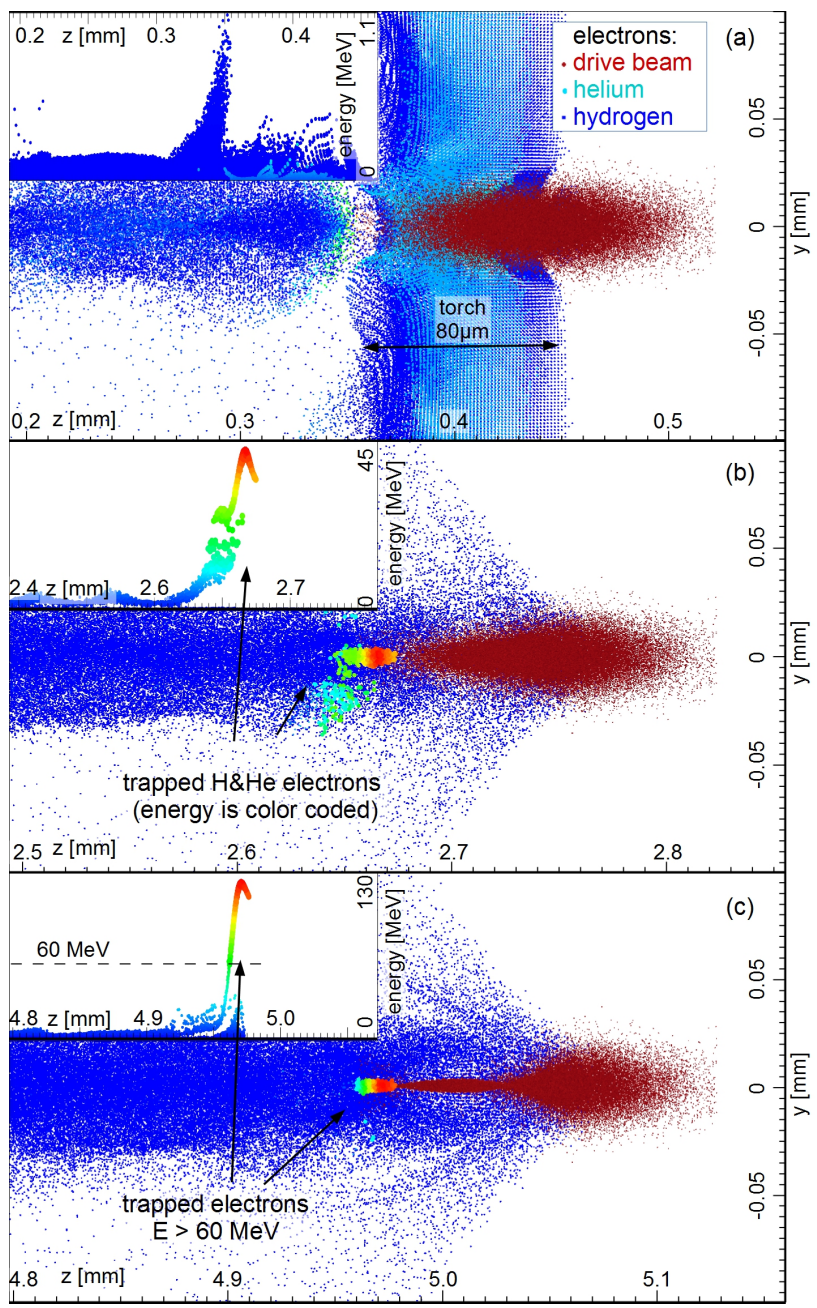

Figure 2: Optical plasma torch injection, illustrated by a central slice of a full $3 \mathrm{~d}$ simulation in scenario (ii), where a hydrogen density of $n_{\mathrm{H}}=4 \times 10^{17} \mathrm{~cm}^{-3}$, and helium density of $n_{\mathrm{He}}=3 \times 10^{17} \mathrm{~cm}^{-3}$ was used (y,z are the torch laser, and drive beam propagation axes). Same as in scenario (i), but in addition the plasma torch also comprises helium electrons (light blue spheres), which leads to a double trapezoidal density shape. The plasma torch is generated in the path of the drive beam, where the laser intensity has been adjusted to ionize helium as well. A laser pulse $w_{0}=35 \mu \mathrm{m}$ and $a_{0}=0.03$ is used. In (a) the torch is crossed, leading to blowout amplification and injection. The injected electrons are shown in $(b) \approx 2.2 \mathrm{~mm}$ behind the torch, and (c) after $\mathrm{z} \approx 4.6 \mathrm{~mm}$ of acceleration, where the maximum energy of $\mathrm{E} \approx 128 \mathrm{MeV}$ is reached.

nario is illustrated, which applies preionized hydrogen (LIT). Using the same plasma densities as in cases (i) and (ii), uncontrolled electron injection would occur due to strong electric fields of the blowout $[10,21,15]$ that would partly ionize and trap helium. To mitigate this effect, reduced gas densities for hydrogen and helium are used: $n_{\mathrm{H}}=5 \times 10^{16} \mathrm{~cm}^{-3}$, and $n_{\mathrm{He}}=1 \times 10^{17} \mathrm{~cm}^{-3}$, since the longitudinal fields are proportional to the ambient plasma density $E_{\mathrm{z}} \propto n^{1 / 2}$. The reduction of the plasma density decreases the electric field, avoiding dark current generation at the rear of the blowout. It shall be noted that alternatively, one may also use a weaker driver bunch 
but elevated hydrogen densities. This would generate smaller 206 blowouts, but nevertheless small enough wakefields as required to avoid He ionization or, more importantly, unwanted trapping. ${ }^{207}$ In this scenario, electron bunch injection and trapping is be- ${ }^{208}$ cause of the extension of the plasma wavelength when leav-209 ing the plasma torch, resulting in electron injection due to the ${ }^{210}$ plasma downramp at the end: a process similar to standard gas ${ }^{211}$ density downramp injection. Obtained witness bunch param- ${ }^{212}$ eters after $\approx 7.7 \mathrm{~mm}$ of acceleration, counting electrons with $^{213}$ energies exceeding $60 \mathrm{MeV}$ are: normalized emittance $\epsilon_{n}=1.8^{214}$ $\mathrm{mm}$ mrad; charge $Q=1.9 \mathrm{nC}$; mean energy at $E=106 \mathrm{MeV} ;{ }^{215}$ energy spread $\sigma_{E} / E=12.6 \%$; and a peak current at $I_{\text {peak }}=22^{216}$ $\mathrm{kA}$.

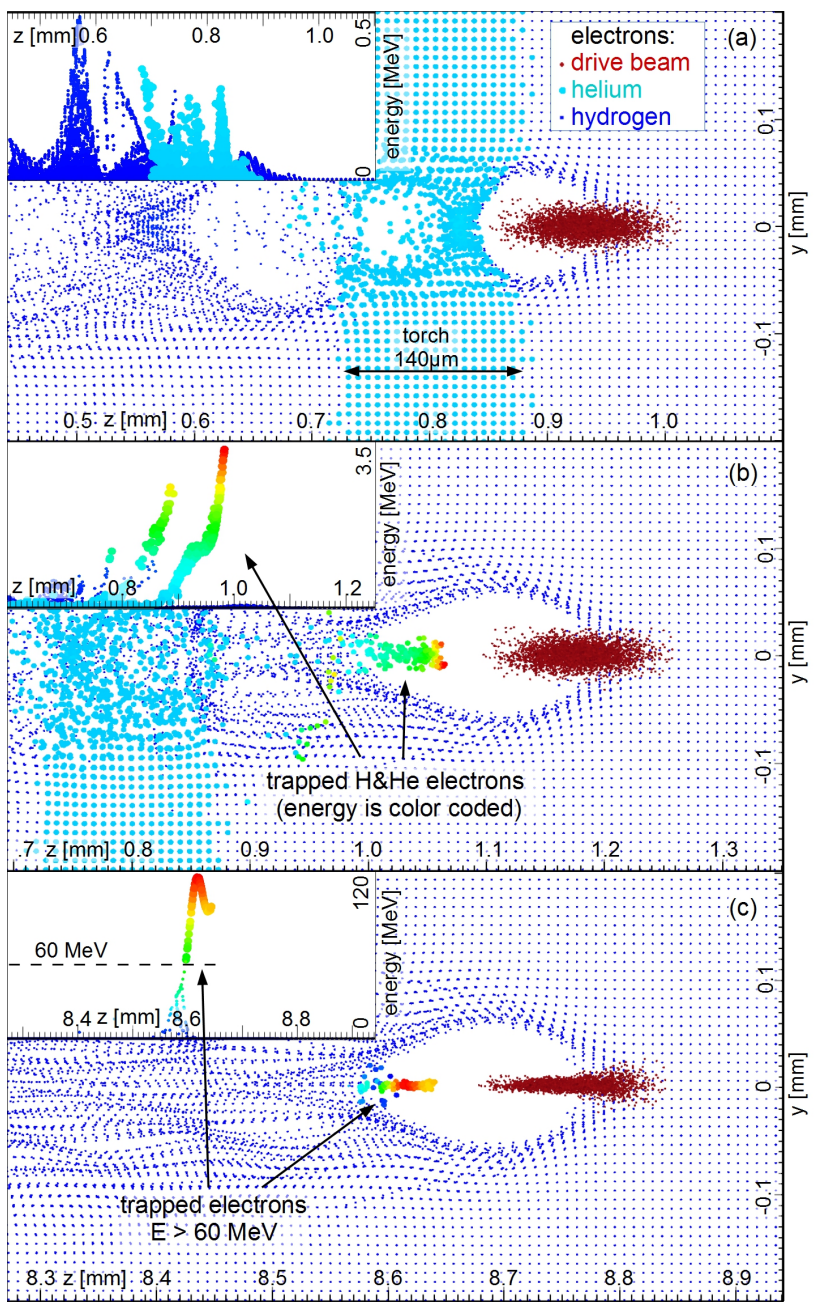
ii and iii). For scenario (iii), an additional laser arm is required

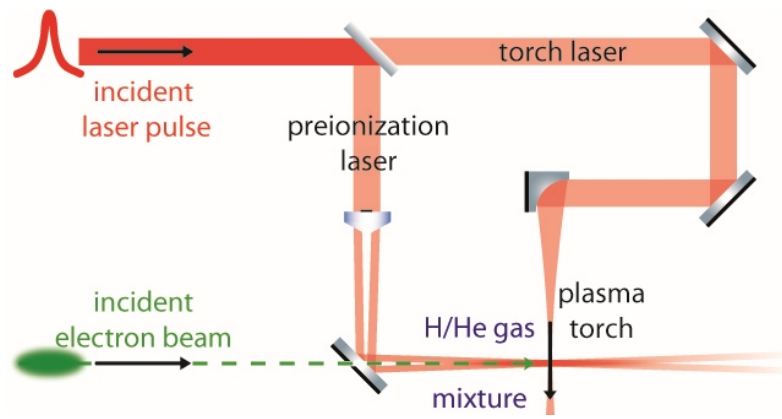

Figure 4: Experimental setup: An electron beam driver and one (scenario i, and ii) or two (scenario iii) moderately synchronized laser pulses interact in an underdense two component gas mixture such as hydrogen and helium. One laser pulse is focused to intensities of the $I \approx 10^{14-15} \mathrm{~W} / \mathrm{cm}^{2}$ level in order to generate the localized hydrogen/helium plasma torch in the path of the electron beam driven blowout. In contrast to the self-ionized scenario (i) and (ii), for scenario (iii) another, high-energy fraction of the laser pulse is used to preionize the hydrogen.
Figure 3: Optical plasma torch injection, illustrated by a central slice of a full $3 \mathrm{~d}$ simulation in scenario (iii) with preionized hydrogen. At gas densities of $n_{\mathrm{H}}=5 \times 10^{16} \mathrm{~cm}^{-3}$, and $n_{\mathrm{He}}=1 \times 10^{17} \mathrm{~cm}^{-3}$, a pronounced blowout is generated. The tunable helium electron torch (a) downramp leads to dark-current ${ }^{234}$ free witness bunch formation (b) and allows for massive charge at substantially235 beam-loaded levels (c), at $124 \mathrm{MeV}$ maximum energy after $\approx 7.7 \mathrm{~mm}$ of ac- -236 celeration. A laser pulse with $w_{0}=100 \mu \mathrm{m}$ and $a_{0}=0.033$ is used to ignite the optical plasma torch of $\approx 140 \mu$ m width (y,z are the torch laser, and beam propagation axes, respectively) which is needed to produce a preionized hydrogen plasma channel around the electron beam axis (or any other means of selective preionization). This general preionization laser needs much higher energy, but as it will be much softer focused than the plasma torch laser (in case of Gaussian focusing optics), or by diffractive optics, will reach much lower peak intensities than the plasma torch laser.

It shall be noted that while the above has been simulated and discussed with respect to electron beam driven PWFA, it could also be used for LWFA. This is not easy for Ti:Sapphire drive pulses, as the high intensities of $I>10^{18} \mathrm{~W} / \mathrm{cm}^{2}$ which are required in order to excite a strong enough bubble, will ionize most media and many higher level ionization states even in the rising slope of the laser pulse driver. However, longer wavelength laser systems such as in the mid-IR and at $\mathrm{CO} 2$ wavelength are showing much progress, and may be intense enough to drive strong wakefields in the future. Such laser pulses, due to their much lower peak electric fields $E=\left(2 \pi m_{e} c^{2} / e\right) a / \lambda(\lambda$ being the laser wavelength, $a$ the normalized amplitude of the laser vector potential), may then allow for plasma density spikes to be generated by short wavelength torch lasers. For example frequency-doubled or tripled Ti:Sapphire laser pulses [19], or even higher harmonics, as the peak electric fields in such laser 
pulses may be much higher when compared to the drive laser 297 pulse while at the same time having much lower ponderomotive ${ }^{298}$ force $F_{p} \simeq-m_{e} c^{2} \Delta a^{2} / 2[30,31]$

\section{Summary}

We have discussed a fully o erate tunable plasma density transitions. The torch width was ${ }^{307}$ chosen to exceed at least one plasma wavelength (within the ${ }_{309}^{308}$ torch) in order to allow the blowout to close within the torch ${ }_{310}$ volume. This defines the ability to trap electrons, e.g. for ${ }^{311}$ high torch densities the plasma wavelength within the torch is ${ }^{312}$ shorter, allowing for a smaller torch width. Different scenarios ${ }_{314}$ have been examined: In scenario (i) injection occurs entirely $y_{315}$ due to the forward and backward shifting of the plasma cavity, ${ }^{316}$ due to the jumping ionization front position and amplification ${ }^{317}$ of the wake. In scenario (iii), where preionized hydrogen is $_{319}$ used, the front of the wakefield does not change with respect tозго the electron beam driver. Here electron injection occurs due to ${ }^{321}$ distortion of the plasma wavelength, which is similar to conven- ${ }^{322}$ tional downramp injection. Scenario (ii) is a mixture of both ${ }_{324}^{323}$ shifting the front of the wakefield combined with a change in 325 the plasma wavelength.

In the optical plasma torch technique a large range of field ${ }_{323}^{327}$ strengths are covered by the accelerating electrons, which re- ${ }_{329}$ sults in a rather large energy spread. However, by using a a $^{30}$ second component (scenario ii and iii) the amount of trapped ${ }^{331}$ charge and the bunch length can be controlled, as well as the ${ }_{333}^{332}$ trapping position. Therefore beam loading can be utilized, e.g. .334 by adjusting the shape of the downramp, to lower the energy ${ }^{335}$ spread along with the possibility to trap very short bunches in ${ }^{336}$ the very rear of the blowout, leaving space for optimization of ${ }_{338}^{337}$ this technique. Additionally different laser profiles and intensi-ззя ties can be applied to create diverse plasma profiles and ramp ${ }^{340}$ lengths, to tune the current profile and length of the witness ${ }^{341}$ bunch and to further optimize the bunch quality. Asymmetric ${ }_{343}^{342}$ plasma torch profiles and multiple plasma torches may allow 344 for further enhanced flexibility.

This work was supported by DFG, STFC 4070022104, $\mathrm{DOE}_{348}^{347}$ DE-SC0009533, DE-FG02-07ER46272, DE-FG03- 92ER40693 by ONR N00014-06-1-0925 and Helmholtz VH-VI-503. We ${ }^{350}$ acknowledge the assistance of the VSim (Tech-X) development ${ }^{351}$ team. This research used computational resources of the $\mathrm{Na}_{-353}$ tional Energy Research Scientific Computing Center, which is ${ }^{354}$ supported by DOE DE-AC02-05CH11231. The authors grate- ${ }^{355}$ fully acknowledge the computing time granted by the John von ${ }_{357}^{356}$ Neumann Institute for Computing (NIC) and provided on the $\mathrm{S}_{358}$ supercomputer JURECA at Juelich Supercomputing Centre (JS 55 , and by the "Norddeutsche Verbund fuer Hoch- und Hoestleis- ${ }^{360}$ tungsrechnen" (HLRN). D. A. J. acknowledges support of the ${ }_{362}^{361}$ UK EPSRC (EP/J018171/1) and the ECs 7th Framework Programme (LASERLAB-EUROPE No. 284464, EUCARD-2 Projeet No. 312453) and the Extreme Light Infrastructure (ELI).
[1] G. Wittig, O. Karger, A. Knetsch, Y. Xi, A. Deng, J. B. Rosenzweig, D. L. Bruhwiler, J. Smith, G. G. Manahan, Z.-M. Sheng, D. A. Jaroszynski, B. Hidding, Optical plasma torch electron bunch generation in plasma wakefield accelerators, Phys. Rev. ST Accel. Beams 18 (2015) 081304, doi: \bibinfo\{doi\}\{10.1103/PhysRevSTAB.18.081304\}, URL http:// link.aps.org/doi/10.1103/PhysRevSTAB.18.081304.

[2] S. Bulanov, N. Naumova, F. Pegoraro, J. Sakai, Particle injection into the wave acceleration phase due to nonlinear wake wave breaking, Phys. Rev. E 58 (1998) R5257-R5260, doi: \bibinfo\{doi\}\{10.1103/PhysRevE. 58.R5257\}, URL http://link.aps.org/doi/10.1103/PhysRevE. 58. R5257.

[3] H. Suk, N. Barov, J. B. Rosenzweig, E. Esarey, Plasma Electron Trapping and Acceleration in a Plasma Wake Field Using a Density Transition, Phys. Rev. Lett. 86 (2001) 1011-1014, doi:\bibinfo\{doi\}\{10.1103/ PhysRevLett.86.1011\}, URL http://link.aps.org/doi/10.1103/ PhysRevLett.86.1011.

[4] C. G. R. Geddes, K. Nakamura, G. R. Plateau, C. Toth, E. CormierMichel, E. Esarey, C. B. Schroeder, J. R. Cary, W. P. Leemans, PlasmaDensity-Gradient Injection of Low Absolute-Momentum-Spread Electron Bunches, Phys. Rev. Lett. 100 (21) (2008) 215004, doi: \bibinfo\{doi\}\{10. 1103/PhysRevLett.100.215004\}.

[5] J. Faure, C. Rechatin, O. Lundh, L. Ammoura, V. Malka, Injection and acceleration of quasimonoenergetic relativistic electron beams using density gradients at the edges of a plasma channel, Physics of Plasmas 17 (8) 083107, doi:〈bibinfo\{doi\}\{http://dx.doi.org/10. 1063/1.3469581\}, URL http: //scitation. aip.org/content/aip/ journal/pop/17/8/10.1063/1.3469581.

[6] K. Schmid, A. Buck, C. M. S. Sears, J. M. Mikhailova, R. Tautz, D. Herrmann, M. Geissler, F. Krausz, L. Veisz, Density-transition based electron injector for laser driven wakefield accelerators, Phys. Rev. ST Accel. Beams 13 (9) (2010) 091301, doi:\bibinfo\{doi\}\{10.1103/PhysRevSTAB. 13.091301\}

[7] A. J. Gonsalves, K. Nakamura, C. Lin, D. Panasenko, S. Shiraishi, T. Sokollik, C. Benedetti, C. B. Schroeder, C. G. R. Geddes, J. van Tilborg, J. Osterhoff, E. Esarey, C. Toth, W. P. Leemans, Tunable laser plasma accelerator based on longitudinal density tailoring, Nat Phys advance online publication (2011) 862-866, URL http://dx.doi.org/ 10.1038/nphys2071.

[8] M. Chen, Z.-M. Sheng, Y.-Y. Ma, J. Zhang, Electron injection and trapping in a laser wakefield by field ionization to high-charge states of gases, Journal of Applied Physics 99 (5) 056109, doi: $\backslash$ bibinfo\{doi\}\{http: //dx.doi.org/10.1063/1.2179194\}, URL http://scitation.aip.org/ content/aip/journal/jap/99/5/10.1063/1.2179194.

[9] D. Umstadter, J. K. Kim, E. Dodd, Laser Injection of Ultrashort Electron Pulses into Wakefield Plasma Waves, Phys. Rev. Lett. 76 (1996) 20732076, doi:\bibinfo\{doi\}\{10.1103/PhysRevLett.76.2073\}, URL http:// link. aps.org/doi/10.1103/PhysRevLett.76.2073.

[10] E. Oz, S. Deng, T. Katsouleas, P. Muggli, C. D. Barnes, I. Blumenfeld, F. J. Decker, P. Emma, M. J. Hogan, R. Ischebeck, R. H. Iverson, N. Kirby, P. Krejcik, C. O'Connell, R. H. Siemann, D. Walz, D. Auerbach, C. E. Clayton, C. Huang, D. K. Johnson, C. Joshi, W. Lu, K. A. Marsh, W. B. Mori, M. Zhou, Ionization-Induced Electron Trapping in Ultrarelativistic Plasma Wakes, Physical Review Letters 98 (8) 084801, doi: \bibinfo\{doi\}\{10.1103/PhysRevLett.98.084801\}, URL http://link.aps.org/abstract/PRL/v98/e084801.

[11] C. McGuffey, A. G. R. Thomas, W. Schumaker, T. Matsuoka, V. Chvykov, F. J. Dollar, G. Kalintchenko, V. Yanovsky, A. Maksimchuk, K. Krushelnick, V. Y. Bychenkov, I. V. Glazyrin, A. V. Karpeev, Ionization Induced Trapping in a Laser Wakefield Accelerator, Phys. Rev. Lett. 104 (2) (2010) 025004, doi: \bibinfo\{doi\}\{10.1103/PhysRevLett.104.025004\}.

[12] A. Pak, K. A. Marsh, S. F. Martins, W. Lu, W. B. Mori, C. Joshi, Injection and Trapping of Tunnel-Ionized Electrons into Laser-Produced Wakes, Phys. Rev. Lett. 104 (2010) 025003, doi: \bibinfo\{doi\}\{10. 1103/PhysRevLett.104.025003\}, URL http://link.aps.org/doi/ 10.1103/PhysRevLett. 104.025003.

[13] C. E. Clayton, J. E. Ralph, F. Albert, R. A. Fonseca, S. H. Glenzer, C. Joshi, W. Lu, K. A. Marsh, S. F. Martins, W. B. Mori, A. Pak, F. S. Tsung, B. B. Pollock, J. S. Ross, L. O. Silva, D. H. Froula, Self-Guided Laser Wakefield Acceleration beyond $1 \mathrm{GeV}$ Using Ionization-Induced Injection, Phys. Rev. Lett. 105 (10) (2010) 105003, doi: bibinfo\{doi\}\{10. 1103/PhysRevLett.105.105003\}. 
14] M. Chen, E. Esarey, C. B. Schroeder, C. G. R. Geddes, W. P. Lee-439 mans, Theory of ionization-induced trapping in laser-plasma accelerators, 440 Physics of Plasmas (1994-present) 19 (3) 033101.

[15] A. Martinez de la Ossa, J. Grebenyuk, T. Mehrling, L. Schaper, J. Os-442 terhoff, High-Quality Electron Beams from Beam-Driven Plasma Accel-443 erators by Wakefield-Induced Ionization Injection, Phys. Rev. Lett. 11144 (2013) 245003, doi: \bibinfo\{doi\}\{10.1103/PhysRevLett.111.245003\},445 URL http://link.aps.org/doi/10.1103/PhysRevLett.111.446 245003.

16] N. Bourgeois, J. Cowley, S. M. Hooker, Two-Pulse Ionization Injec-4 tion into Quasilinear Laser Wakefields, Phys. Rev. Lett. 111 (2013)449 155004, doi: \bibinfo\{doi\}\{10.1103/PhysRevLett.111.155004\}, URL450 http://link.aps.org/doi/10.1103/PhysRevLett.111.155004. 45

17] B. Hidding, G. Pretzler, D. Bruhwiler, J. Rosenzweig, Method for gen-452 erating electron beams in a hybrid plasma accelerator, german Patent DE453 102011104 858.1, US/PCT patent Ser. No. PCT/US12/043002, 2011. 454

[18] B. Hidding, G. Pretzler, J. B. Rosenzweig, T. Königstein, D. Schiller,45 D. L. Bruhwiler, Ultracold Electron Bunch Generation via Plasma456 Photocathode Emission and Acceleration in a Beam-Driven Plasma457 Blowout, Phys. Rev. Lett. 108 (2012) 035001, doi: $\backslash$ bibinfo\{doi $\}\{10.458$ 1103/PhysRevLett.108.035001\}, URL http://link.aps.org/doi/459 10.1103/PhysRevLett.108.035001.

[19] B. Hidding, J. B. Rosenzweig, Y. Xi, B. O’Shea, G. Andonian, D. Schiller, S. Barber, O. Williams, G. Pretzler, T. Königstein, F. Kleeschulte, M. J. Hogan, M. Litos, S. Corde, W. W. White, P. Muggli, D. L. Bruhwiler, K. Lotov, Beyond injection: Trojan horse underdense photocathode plasma wakefield acceleration, AIP Conference Proceedings 1507 (1) (2012) 570-575, doi:\bibinfo\{doi\}\{10.1063/1.4773760\}, URL http:// link. aip.org/link/?APC/1507/570/1.

[20] Y. Xi, B. Hidding, D. Bruhwiler, G. Pretzler, J. B. Rosenzweig, Hybrid modeling of relativistic underdense plasma photocathode injectors, Phys. Rev. ST Accel. Beams 16 (2013) 031303, doi: \bibinfo\{doi $\}\{10$. 1103/PhysRevSTAB.16.031303\}, URL http://link.aps.org/doi/ 10.1103/PhysRevSTAB. 16.031303.

[21] F. Li, J. F. Hua, X. L. Xu, C. J. Zhang, L. X. Yan, Y. C. Du, W. H. Huang, H. B. Chen, C. X. Tang, W. Lu, C. Joshi, W. B. Mori, Y. Q. Gu, Generating High-Brightness Electron Beams via Ionization Injection by Transverse Colliding Lasers in a Plasma-Wakefield Accelerator, Phys. Rev. Lett. 111 (2013) 015003, doi:\bibinfo\{doi\}\{10 1103/PhysRevLett.111.015003\}, URL http://link.aps.org/doi/ 10.1103/PhysRevLett.111.015003.

[22] X. L. Xu, et al., Phase-Space Dynamics of Ionization Injection in Plasma-Based Accelerators, Phys. Rev. Lett. 112 (2014) 035003, doi: |bibinfo\{doi\}\{10.1103/PhysRevLett.112.035003\}, URL http://link. aps.org/doi/10.1103/PhysRevLett.112.035003.

[23] P. Muggli, S. Lee, T. Katsouleas, R. Assmann, F. J. Decker, M. J. Hogan, R. Iverson, P. Raimondi, R. H. Siemann, D. Walz, B. Blue, C. E. Clayton, E. Dodd, R. A. Fonseca, R. Hemker, C. Joshi, K. A. Marsh, W. B. Mori, S. Wang, Collective refraction of a beam of electrons at a plasma-gas interface, Phys. Rev. ST Accel. Beams 4 (2001) 091301, doi: bibinfo\{doi\}\{10.1103/PhysRevSTAB.4.091301\}, URL http://link. aps.org/doi/10.1103/PhysRevSTAB .4.091301.

[24] T.-Y. Chien, C.-L. Chang, C.-H. Lee, J.-Y. Lin, J. Wang, S.Y. Chen, Spatially Localized Self-Injection of Electrons in a SelfModulated Laser-Wakefield Accelerator by Using a Laser-Induced Transient Density Ramp, Phys. Rev. Lett. 94 (2005) 115003, doi: |bibinfo\{doi\}\{10.1103/PhysRevLett.94.115003\}, URL http://link. aps.org/doi/10.1103/PhysRevLett.94.115003.

[25] P. Brijesh, C. Thaury, K. T. Phuoc, S. Corde, G. Lambert, V. Malka, S. P. D. Mangles, M. Bloom, S. Kneip, Tuning the electron energy by controlling the density perturbation position in laser plasma accelerators, Physics of Plasmas 19 (6) 063104, doi: \bibinfo\{doi\}\{http://dx.doi.org/10. 1063/1.4725421\}, URL http://scitation.aip.org/content/aip/ journal/pop/19/6/10.1063/1.4725421.

[26] C. Nieter, J. R. Cary, VORPAL: a versatile plasma simulation code, Journal of Computational Physics 196 (2) (2004) 448-473, ISSN 00219991, URL http://www.sciencedirect.com/science/article/ pii/S0021999103006041.

[27] M. Litos, E. Adli, W. An, C. I. Clarke, C. E. Clayton, S. Corde, J. P. Delahaye, R. J. England, A. S. Fisher, J. Frederico, S. Gessner, S. Z. Green, M. J. Hogan, C. Joshi, W. Lu, K. A. Marsh, W. B. Mori, P. Mug- gli, N. Vafaei-Najafabadi, D. Walz, G. White, Z. Wu, V. Yakimenko, G. Yocky, High-efficiency acceleration of an electron beam in a plasma wakefield accelerator, Nature 515 (7525) (2014) 92-95, ISSN 0028-0836, URL http://dx.doi.org/10.1038/nature13882.

[28] D. L. Bruhwiler, D. A. Dimitrov, J. R. Cary, E. Esarey, W. Leemans, R. E. Giacone, Particle-in-cell simulations of tunneling ionization effects in plasma-based accelerators, Physics of Plasmas (1994present) 10 (5) (2003) 2022-2030, doi:\bibinfo\{doi\}\{http://dx.doi.org/10. 1063/1.1566027\}, URL http: //scitation.aip.org/content/aip/ journal/pop/10/5/10.1063/1.1566027.

[29] H. Suk, N. Barov, J. B. Rosenzweig, E. Esarey, Plasma Electron Trapping and Acceleration in a Plasma Wake Field Using a Density Transition, Phys. Rev. Lett. 86 (6) (2001) 1011-1014, URL http://link. aps.org/doi/10.1103/PhysRevLett.86.1011.

[30] D. Umstadter, J.-K. Kim, E. Dodd, Method and apparatus for generating and accelerating ultrashort electron pulses, uS patent Ser. No. 5,789,876, 1995.

[31] L.-L. Yu, E. Esarey, C. B. Schroeder, J.-L. Vay, C. Benedetti, C. G. R. Geddes, M. Chen, W. P. Leemans, Two-Color Laser-Ionization Injection, Phys. Rev. Lett. 112 (2014) 125001, doi: \bibinfo\{doi\}\{10. 1103/PhysRevLett.112.125001\}, URL http://link.aps.org/doi/ 10.1103/PhysRevLett.112.125001. 